\title{
Genome-wide association study reveals a patatin-like lipase relating to the reduction of seed oil content in Brassica napus
}

\author{
Haoyi Wang ${ }^{1}$, Qian Wang ${ }^{1}$, Haksong Pak', Tao Yan¹, Mingxun Chen², Xiaoyang Chen, Dezhi Wu and \\ Lixi Jiang ${ }^{1 *}$ (D)
}

\begin{abstract}
Background: Rapeseed (Brassica napus L.) is an important oil crop world-widely cultivated, and seed oil content $(\mathrm{SOC})$ is one of the most important traits for rapeseed. To increase SOC, many efforts for promoting the function of genes on lipid biosynthesis pathway have been previously made. However, seed oil formation is a dynamic balance between lipid synthesis and breakdown. It is, therefore, also reasonable to weaken or eliminate the function of genes involved in lipid degradation for a higher final SOC.

Results: We applied a genome-wide association study (GWAS) on SOC in a collection of 290 core germplasm accessions. A total of 2,705,480 high-quality SNPs were used in the GWAS, and we identified BnaC07g30920D, a patatin-like lipase (PTL) gene, that was associated with SOC. In particular, six single-nucleotide-polymorphisms (SNPs) in the promoter region of BnaC07g30920D were associated with the significant reduction of SOC, leading to a 4.7$6.2 \%$ reduction of SOCs. We performed in silico analysis to show a total of 40 PTLs, which were divided into four clades, evenly distributed on the A and C subgenomes of Brassica napus. RNA-seq analysis unveiled that BnPTLs were preferentially expressed in reproductive tissues especially maturing seeds.

Conclusions: We identified BnaC07g30920D, a BnPTL gene, that was associated with SOC using GWAS and performed in silico analysis of 40 PTLs in Brassica napus. The results enrich our knowledge about the SOC formation in rapeseed and facilitate the future study in functional characterization of BnPTL genes.
\end{abstract}

Keywords: Seed oil content, Lipase, Patatin, GWAS, Rapeseed, Lipid degradation, RNA-seq analysis

\section{Background}

Rapeseed (Brassica napus L., AACC, $2 \mathrm{n}=38$ ) is the third-largest source of vegetable oil in the world after soybean and palm [1]. Seed oil content (SOC) is one of the most important traits in rapeseed. Overall, the SOC among rapeseed varieties varies from 26 to $50 \%$ [2]. It is an important goal for rapeseed breeders to have varieties with high SOC.

\footnotetext{
* Correspondence: jianglx@zju.edu.cn

${ }^{1}$ Institute of Crop Science, Zhejiang University, Yu-Hang-Tang Road 866, Hangzhou 310058, China

Full list of author information is available at the end of the article
}

Seed oil accumulation is a dynamic balance between lipid biosynthesis and breakdown. Lipid biosynthesis in plant seeds is activated after seed embryo formation and it involves a series of pathways, such as carbohydrate metabolism, fatty acids (FAs) elongation, triacylglycerol (TAG) synthesis and oil-body formation [3]. In brief, the synthesis of lipids in seeds can be roughly divided into three stages. First, those 16C-18C FAs including palmitic acid (C16:0), stearic acid (C18:0), and oleic acid (C18:1) are synthesized in plastids using pyruvate from glycolysis as the primary substrate. Second, the 16C-18C FAs are transported to the endoplasmic reticulum, where they

(c) The Author(s). 2020 Open Access This article is licensed under a Creative Commons Attribution 4.0 International License, which permits use, sharing, adaptation, distribution and reproduction in any medium or format, as long as you give appropriate credit to the original author(s) and the source, provide a link to the Creative Commons licence, and indicate if changes were made. The images or other third party material in this article are included in the article's Creative Commons licence, unless indicated otherwise in a credit line to the material. If material is not included in the article's Creative Commons licence and your intended use is not permitted by statutory regulation or exceeds the permitted use, you will need to obtain permission directly from the copyright holder. To view a copy of this licence, visit http://creativecommons.org/licenses/by/4.0/. The Creative Commons Public Domain Dedication waiver (http://creativecommons.org/publicdomain/zero/1.0/) applies to the data made available in this article, unless otherwise stated in a credit line to the data. 
are catalyzed by a series of enzymes for the elongation and desaturation of the carbon chains. Finally, TAGs are synthesized based on FAs and glycerol, and stored in 1$2 \mu \mathrm{m}$ oil bodies [3-8]. The transcription factors (TFs) such as LEAFY COTYLEDON1 (LEC1), LEC2, ABA INSE NSITIVE3, FUSCA3 and WRINKLED1 are key regulators of seed FA biosynthesis and are expressed in seeds. Their expressions overlap various developmental stages $[6,9]$.

The accumulation of lipids in rapeseed is not a unilateral synthesis process but a dynamic balance between anabolism and catabolism. Seed lipids reduce at the late stage of seed maturation at an approximate rate of $10 \%$ on average [10]. Oil degradation is catalyzed by a variety of lipases that hydrolyze TAG and set FAs free [11]. The free FAs are unstable and subjected to further degradation for acetyl-CoA in a process known as beta-oxidation taking place in glyoxysomes. The acetyl-CoA is further degraded into 4-carbon compounds in the so-called glyoxylate cycle occurring in peroxisomes [1,12-14].

It is estimated that the reduction of SOC at the late maturation stage of rapeseed results in a loss of about 20 million tons of oil per year [10]. A previous study revealed that five genes, namely BnSFAR1 to BnSFAR5 belonging to the GDSL family are involved in the decomposition of lipids in developing seeds of rapeseed, and thus reduced SOC [15]. However, GDSL lipase could not be the only type of lipase that play roles in seed lipid decomposition.

Patatin is a single lipolytic acyl-hydrolase with broad substrate specificity, firstly found in potato tubers in 1970 [16]. Instead of GXSXG or GDSL motif, the active site of the patatin domain consists of a distinct Ser-Asp catalytic dyad [17]. Studies indicated that plant patatin-related enzymes are involved in diverse biofunctions, including plant responses to auxin, elicitors or pathogens, and abiotic stresses and lipid mobilization during seed germination [18-21]. Many patatin-like lipases (PTLs) such as SUGAR-DEPEND ENT1 (SDP1) act as phospholipases or TAG lipases $[18,22,23]$. The functions of most PTLs remain little understood.

In the past decades, quantitative trait locus (QTL) mapping unearthed plenty of information on the molecular mechanism controlling the formation of total FAs [24, 25] and FA species [26, 27] in rapeseed. More recently, genome-wide association studies (GWAS) combined with linkage disequilibrium (LD) mapping emerged as a more effective tool in identifying candidate genes that control agronomic and quality traits in a large genetic population $[28,29]$. The rapid development of genome sequencing technologies enabled the identification of single nucleotide polymorphisms (SNPs) in a huge quantity [28-32]. LD mapping which indicates the degree of correlation between significant SNPs and candidate genes is a population-based survey technology for a precise QTL localization [28, 29, 33-35].

To better understand the genetic control of SOC in a polyploid genome of rapeseed, we performed GWAS on SOC in a collection of 290 core germplasm accessions representing a total of 991 genetic accessions originated from 39 countries of the world. We identified BnaC07g30920D, a BnPTL gene, that was significantly associated with SOC. We analyzed the expression of Bna.C07.PTL in various tissues, and performed in silico analysis to show an overall distribution of the PTL family in the rapeseed genome.

\section{Results}

\section{Phenotypic variation of SOC}

We measured the SOC of the 290 core accessions (Table S1) at two locations, namely, Changxing (CX) and Jinhua $(\mathrm{JH})$, in 2018 and 2019, respectively. The overall statistics of the SOC were listed in Table 1. The SOC ranged from 29.80 to $54.52 \%$ in CX and from 35.09 to $56.55 \%$ in $\mathrm{JH}$, with an average of 43.25 and $47.98 \%$, respectively (Table 1 ). The coefficient of variations $(\mathrm{CVs})$ for $\mathrm{CX}$ and $\mathrm{JH}$ were nearly identical, indicating a similar degree of SOC variation at these two locations (Table 1). The SOCs in both locations were approximately normal-distributed, and the SOCs of most accessions ranged between 40 and 50\% (Figure S1). Analysis of variance (ANOVA) was performed. It was found that both genotype and environment had significant effects on SOC $(P<0.01)$ (Table 1$)$.

\section{Identification of a BnPTL gene significantly associated with SOC}

Using 2,705,480 SNPs (MAF $>0.05$, missing rate $<0.5$ ), we performed GWAS on SOC in a population including 290 accessions at $\mathrm{CX}$ and $\mathrm{JH}$. The Manhattan Plots show that most significantly associated SNPs $\left(p<10^{-5}\right)$ were mapped on chromosome $\mathrm{C07}$ (Chr.C07) and random chromosome An (Chr.Ann) for the experiment at CX (Figure S2A), and were on Chr.A08 and Chr.C07 for the experiment at $\mathrm{JH}$ (Figure $\mathrm{S} 2 \mathrm{~B}$ ), respectively. The quantile-quantile $(\mathrm{Q}-\mathrm{Q})$ plots showed that there was a significant correlation between SOCs and genotypes by natural selection (Figure S3). In light of the results from both environments, the SNPs located between 35.25 Mbp and $35.79 \mathrm{Mbp}$ on Chr.C07 were taken for further investigation (Figure S2, Table S2). A total of 31 and 22 SNP signals on Chr.C07 were significantly associated with SOC at $\mathrm{CX}$ and $\mathrm{JH}$, respectively (Table S2), suggesting 50 and 34 genes on $\mathrm{Chr} . \mathrm{C07}$ in $\mathrm{CX}$ and $\mathrm{JH}$, respectively, that might be responsible for SOC variations (Table S2). Of these, BnaC07g30920D, annotated as a patatin-like phospholipase, was a putative ortholog of At1g33270 encoding an adiponutrin (ATGL)-like 
Table 1 The overall statistics of phenotypic variation and analysis of variance (ANOVA) of SOC in the 290-accession core collection

\begin{tabular}{lllllllll}
\hline Trait & Locations & Mean \pm SD (\%) & Range (\%) & Skewness & Kurtosis & CV (\%) & Genotype & Environment \\
\hline SOC & CX & $43.25 \pm 2.86$ & $29.80-54.52$ & -0.386 & 2.383 & 6.6 & $* *$ & $*$ \\
& JH & $47.98 \pm 3.21$ & $35.09-56.55$ & -0.876 & 1.81 & 6.7 & & $*$
\end{tabular}

Note: SD standard deviation, CV coefficient of variation, ${ }^{* *}(P<0.01)$

protein. The SNP, ChrC07_35249208, on Chr.C07 was linked $\left(R^{2}=0.68\right)$ with BnaC07g30920D (Fig. 1), indicating their linkage disequilibrium. There were $55 \mathrm{SNPs}$ at BnaC07g30920D, including 51 SNPs located on the 5'end regulatory region, 2 SNPs on the coding sequence (CDS) region, and 2 SNPs within introns (Table S3).

To know the effect of these SNPs on SOC, we performed single SNP association tests for the 55 SNPs among the 290 accessions with different SOCs. The test showed that six SNPs, namely T/G of ChrC07_35,181, 691, C/T of ChrC07_35,181,026, C/T of ChrC07_35,179, 748, A/T of ChrC07_35,179,634, A/G of ChrC07_35,179, 572 and A/G of ChrC07_35,177,247 on the 5'-end regulatory region of BnaC07g30920D were significantly $(p<$ 0.05 ) associated with SOC (Fig. 2). Any nucleotide change in the six positions led to a $4.7-6.2 \%$ reduction of SOCs (Fig. 2). We also measured the effects of other 4 SNPs in the coding sequence and intron regions.
Significant effects were not identified, therefore, we only presented the functional SNPs in Fig. 2. We also showed the effects of these SNPs in Figure S4. In order to identify cis-element in the variation site, we BLAST the nucleotide sequence $5 \mathrm{~Kb}$ upstream to the start codon in the databases PlantProm (http://linux1.softberry.com/berry. phtml?topic=plantprom\&group $=$ data\&subgroup $=$ plantprom) and AGRIS (https://agris-knowledgebase.org/), and identified the SNPs such as T/G (ChrC07_35,181,691) and A/T (ChrC07_35,179,634) locating in the CAAT-box, which associated with SOC changes.

The expression of BnaC07g30920D was analyzed based on two transcriptome datasets (Fig. 3). The highest transcriptional levels of BnaC07g30920D, which were indicated by the number of read counts after calibration, were observed in pistils and sepals (Fig. 3a). BnaC07g30920D was significantly expressed (RPKM $>1)$ in the seeds at 16 DAP, 40 DAP and in germinating seeds 20 HAS (Table S6).

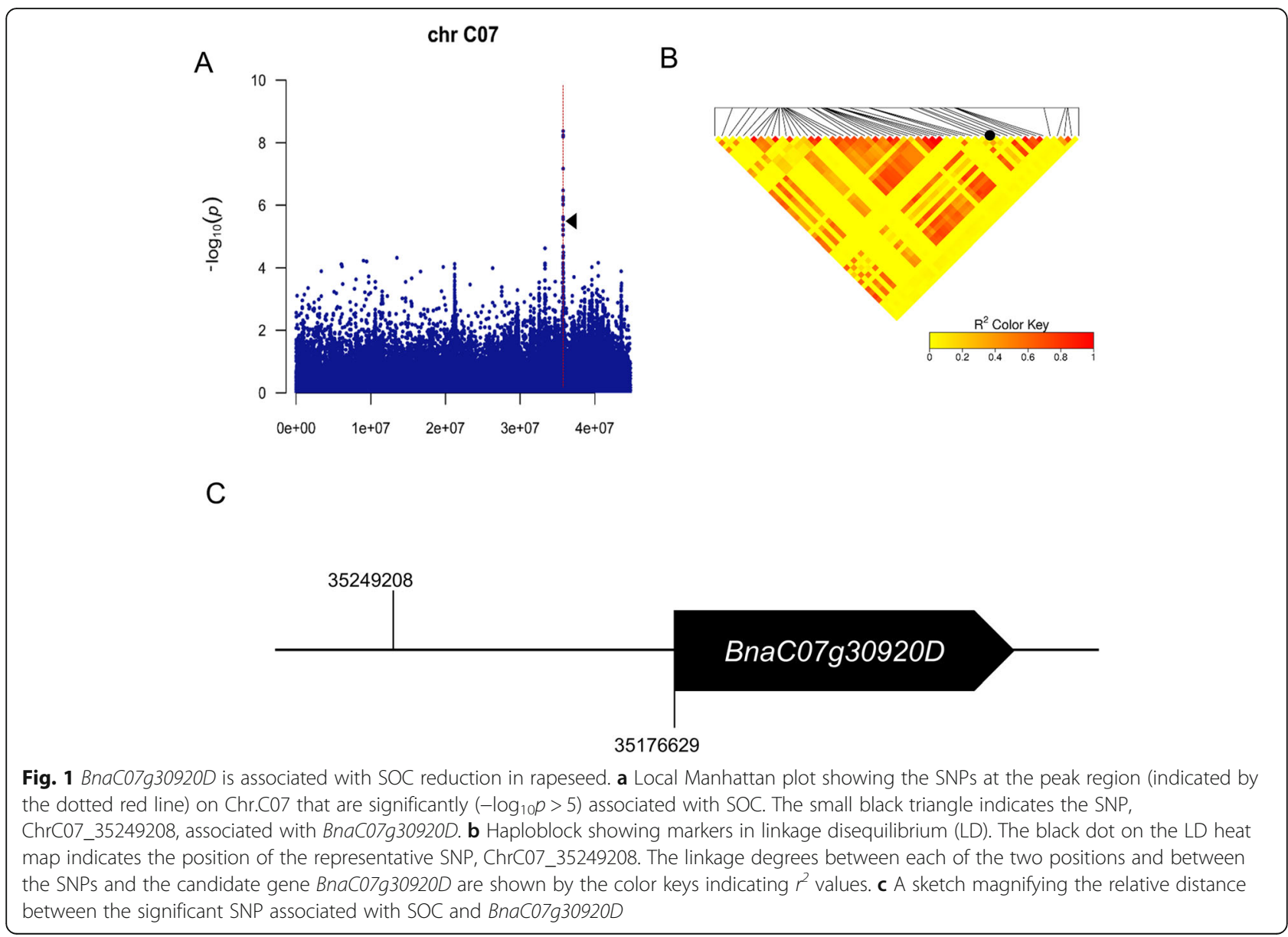




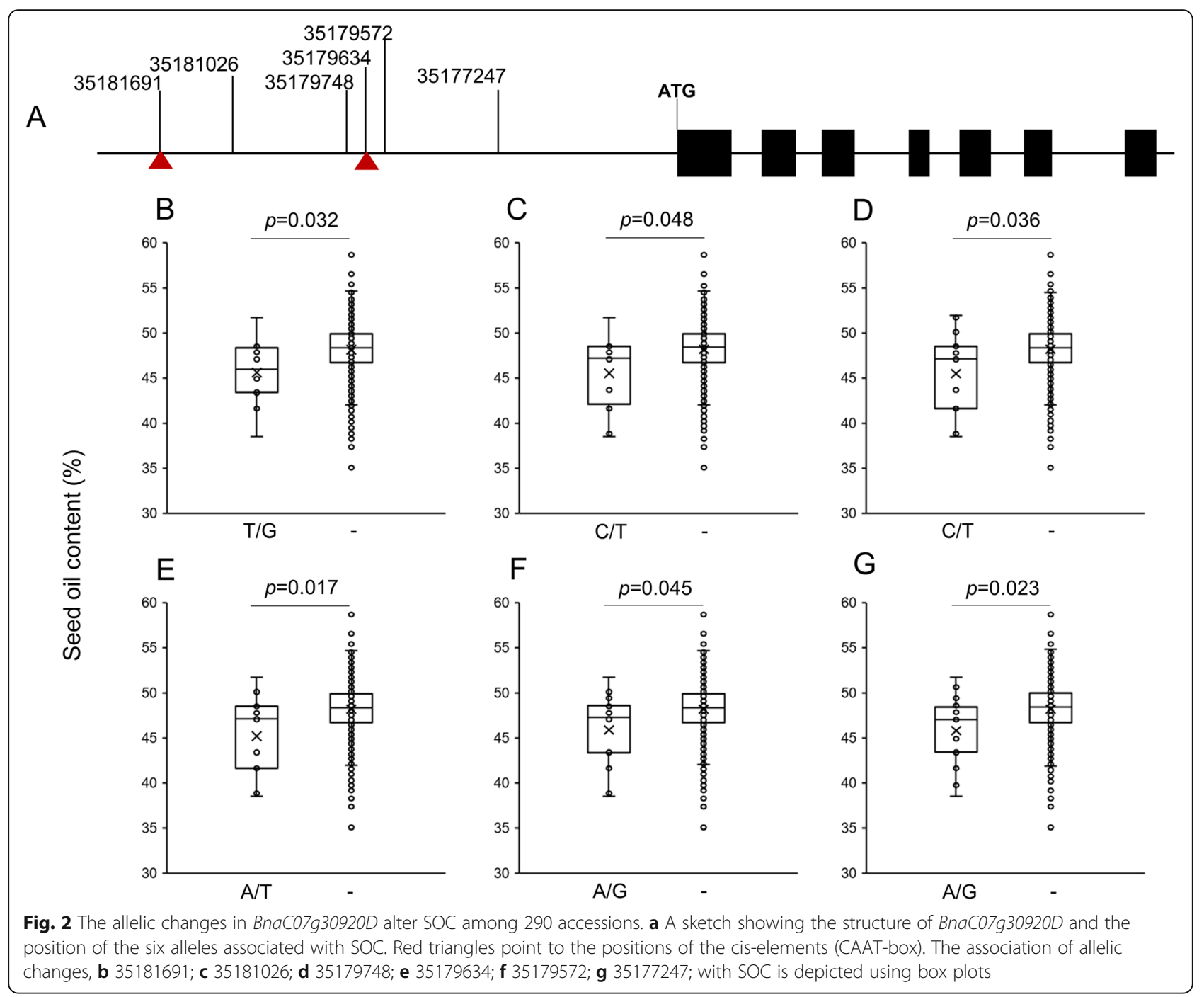

Relative to the 20-HAS germinating seeds, the 16-DAP and 40-DAP seeds had nearly 1.5 folds higher transcriptional level of BnaC07g30920D (Fig. 3b, Table S6), indicating high expression of BnaC07g30920D during seed maturation.

\section{In silico analysis of BnPTLs in rapeseed genome}

Seed oil content is a typical quantitative trait that is controlled by the interaction between numerous major and/or minor genes and environmental factors. In order to excavate potential PTLs that would decompose seed lipid, we performed an in silico analysis to demonstrate the overall distribution of BnPTLs across the rapeseed genome ('Darmor-bzh', V4.1). An InterPro search identified a total of 40 genes containing IPR002641, which were annotated as PTLs. Information such as locus ID, genomic sequence length, coding sequence (CDS) and protein size of putative PTLs as well as the number of their exons and introns were provided in Table S4. The sizes of genomic sequences, CDSs and proteins of the BnPTLs ranged from 1411 to $8211 \mathrm{bp}, 873$ to $4278 \mathrm{bp}$, and 290 to 1425 aa, respectively (Table S4). The 40 BnPTLs were distributed equally on A subgenome and $C$ subgenome (Fig. 4). Except for four BnPTLs (BnaAnng00900D, BnaCnng07580D, BnaCnng10420D and BnaCnng30060D) distributed on random chromosomes, the remaining 36 BnPTLs were mapped on chromosomes A01-A10 and C01-C09. Notably, there were no BnPTLs located on chromosomes A02, A06 and C05 (Fig. 4). There was the largest number of BnPTLs (5 out of 40) located on Chromosome A03, whereas, there was merely one BnPTL homolog which located on chromosomes A04, A05, A08, A10, C02, C06 and C09 (Fig. 4). Nearly half (19 in 40) of BnPTLs located at ends of the chromosomes (Fig. 4).

Gene structure analysis showed that the number of exons of BnPTLs ranged from two to eighteen with 5.6 as average. $12(30 \%)$ and $11(27.5 \%)$ BnPTLs had three and seven exons interrupted by two and six introns, respectively (Table S4, Fig. 5). The rest of the genes had 2 


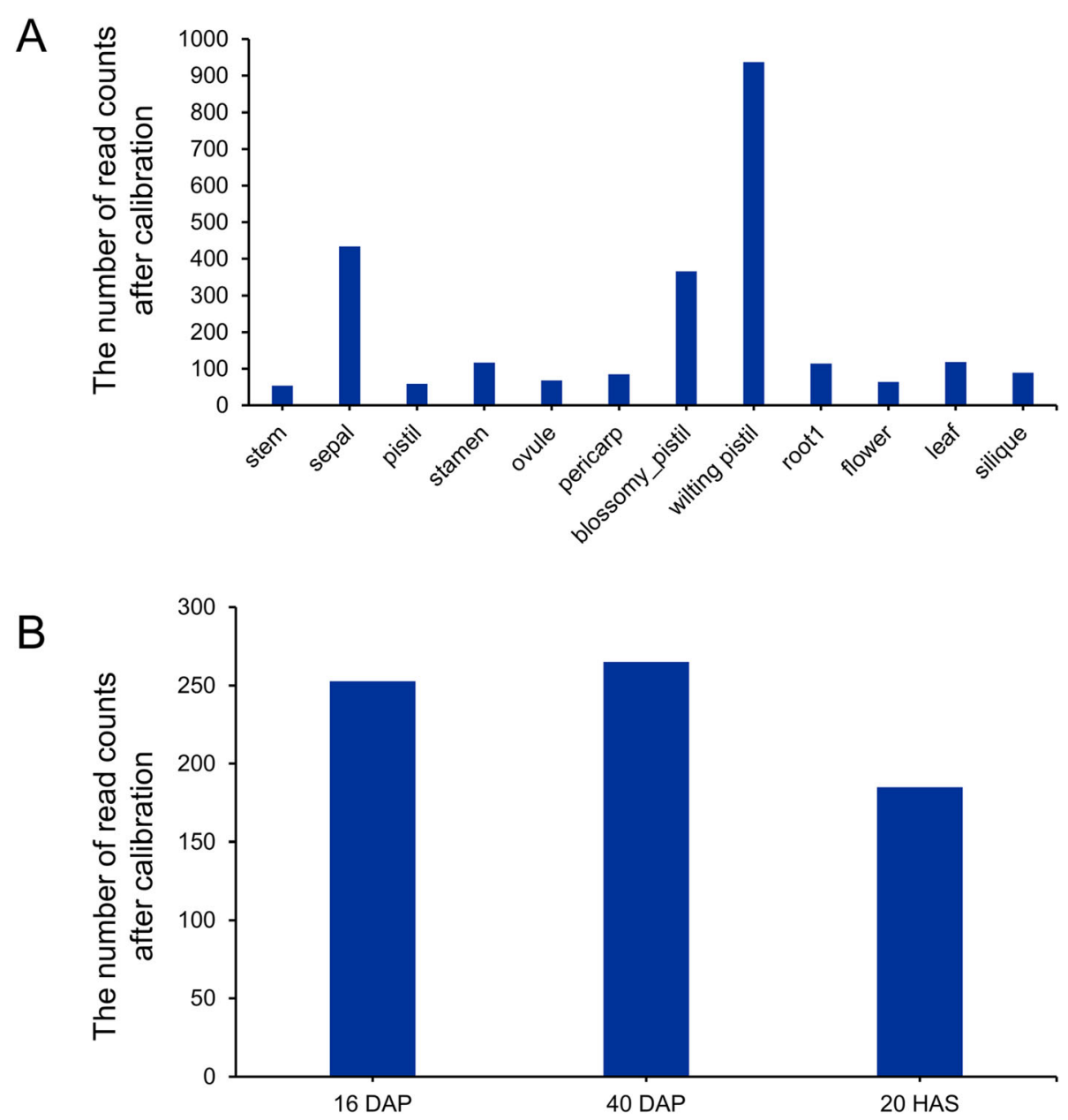

Fig. 3 Expression analysis of BnaC07g30920D. a The expression values of BnaC07g30920D in 12 different tissues. b The expression values of BnaC07g30920D at 16 DAP, 40 DAP of seeds and in germinating seeds 20 HAS

exon (6 genes), 4 exons ( 2 genes), 5 exons (1 gene, BnaA05g06080D), 6 exons (3 genes), 8 exons (1 gene, BnaA10g26130D), 10 exons (1 gene, BnaA08g06610D), 13 exons (1 gene, BnaA03g53720D) or 18 exons (2 genes), respectively.

To elucidate the evolutionary relationship of BnPTLs, we carried out a phylogenetic analysis of BnPTLs. In brief, BnPTLs could be divided into four clades (I-IV) (Fig. 5). The Clade I, Clade II, Clade III and Clade IV contained 14, 12, 8 and 6 BnPTL homologs, accounting for $35,30,20$ and $15 \%$ of the total BnPTLs, respectively (Table S4, Fig. 5). There were significantly more BnPTL exons in genes of Clade II and Clade IV (8.5 on average) than those in Clade I and Clade III (3.1 on average) (Table S4, Fig. 5).

For any gene family, the conserved sequences and domains are related to their functions. In order to better understand the biological function of BnPTLs, we analyzed the conserved domain of BnPTLs. The catalytic regions consisting of DGGGXX, elements for phosphate- or anion-binding, and GXSXG which is the esterase box, were built up with 20-30 amino acids and length of the sequences varied among different clades, suggesting various biological functions of the BnPTL homologs (Table 2).

\section{Expression patterns of BnPTLs}

The expression pattern of BnPTLs can give us additional insight into their biological functions. To determine the expression patterns of BnPTLs in different tissues, transcriptome data from 12 tissues of the B. napus cultivar 'ZS 11' were downloaded [36]. Overall, BnPTLs were expressed in all 12 tissues, implying their diverse biological functions (Fig. 6a, Table S5). The highest expression of BnPTLs was detected in stamens (366 on average) and wilting pistils (362 on average), while the expression of BnPTLs in stems (73 on average), roots (112 on average) and leaves (52 on average) were relatively low, indicating that BnPTLs were expressed preferentially in reproductive tissues. 


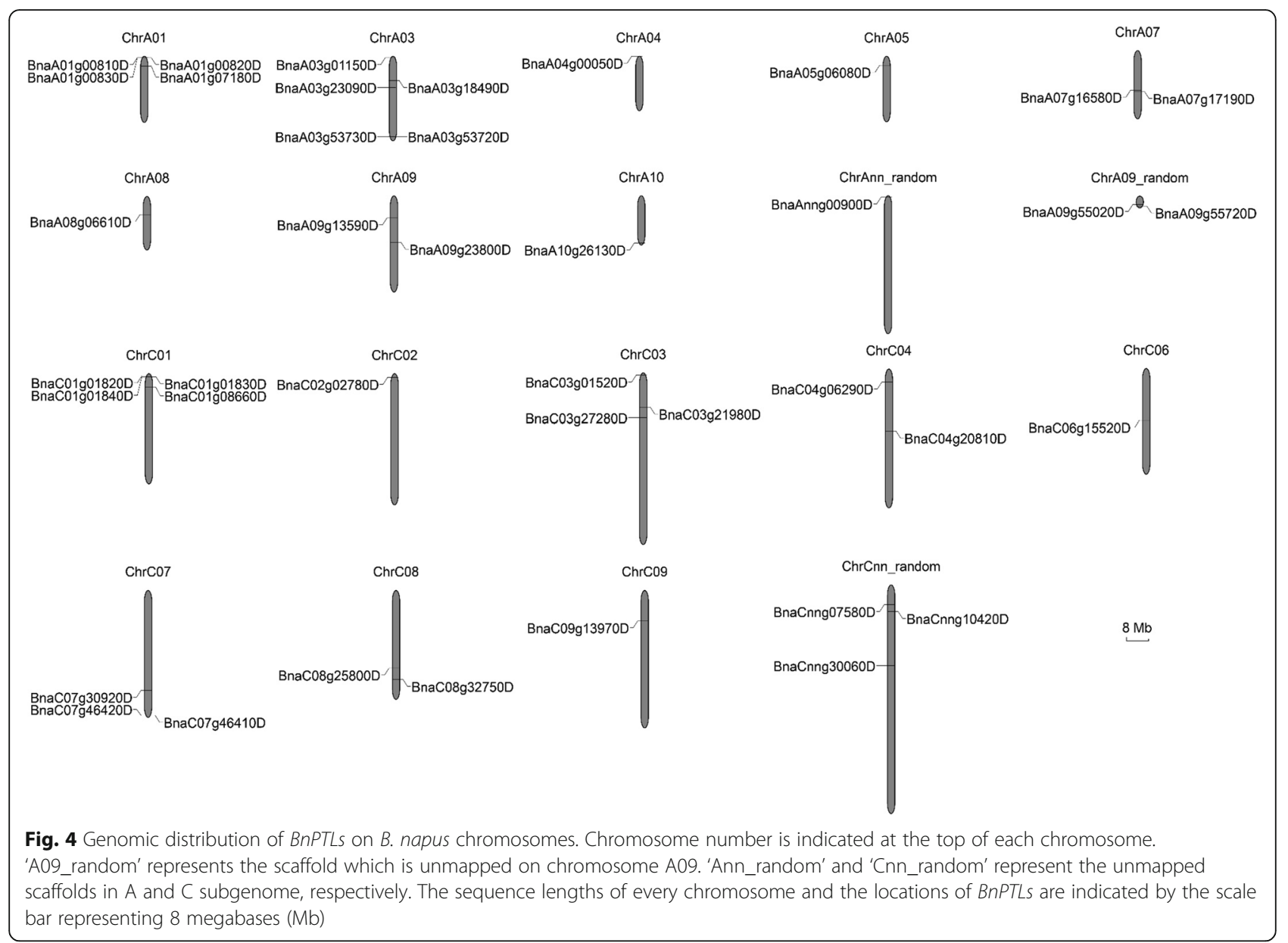

Among 40 BnPTLs, extremely significant expression of some genes such as BnSDP1 (BnaCnng10420D) in wilting pistils (1991) and BnSDP1-Like (BnSDP1L, BnaA07g17190D) in stamens (984), could be observed, suggesting their potential roles in these tissues (Fig. 6a, Table S5).

The BnPTLs which expressed in seeds may affect SOC. To determine the expression pattern of BnPTLs at different developmental stages of seeds, we analyzed the RNA-Seq transcriptome data from seeds of 16, 40 DAP and 20 HAS. There were a total of 31 BnPTLs that were significantly expressed (RPKM $>1$ ) in 40-DAP seeds (Fig. 6, Table S6). In contrast, 18 and 21 BnPTLs were expressed in 16-DAP seeds and the 20-HAS germinating seeds, respectively. Notably, 14 BnPTLs were significantly expressed in seeds of all three developmental stages (Fig. 6b, Table S6). BnaA05g06080D was only expressed in the 16-DAP seeds, whereas, there were seven genes only expressed in 40-DAP seeds. The expression of twelve genes was upregulated in the 40DAP seeds relative to the 16-DAP seeds (defined by $\log 2$ (expression folds) $>1)$. On the other hand, the expression of six genes was downregulated in the 40-DAP seeds relative to the 16-DAP seeds (defined by $\log 2$ (expression folds) $<1$ ) (Fig. 6, Table S6). These results suggested that most BnPTLs had higher expression at the late stage of seed development.

\section{Discussion}

Crop based oils are valuable agricultural products, and SOC is an important trait of oil crops. Elevating SOC was successfully achieved through traditional breeding and genetic manipulation of genes involving lipid biosynthesis [37-39]. In rapeseed, SOC usually first reaches a peak followed by a decline at the late maturity stage [10]. The SOC reduction at the late maturity stage results from two aspects. First, the expression of genes involved in oil synthesis is significantly decreased. Second, a large number of genes involved in oil degradation are expressed and active at this stage [1]. A recent study reported that SOC of rapeseed is affected by the germination potential of premature developing seeds. The 


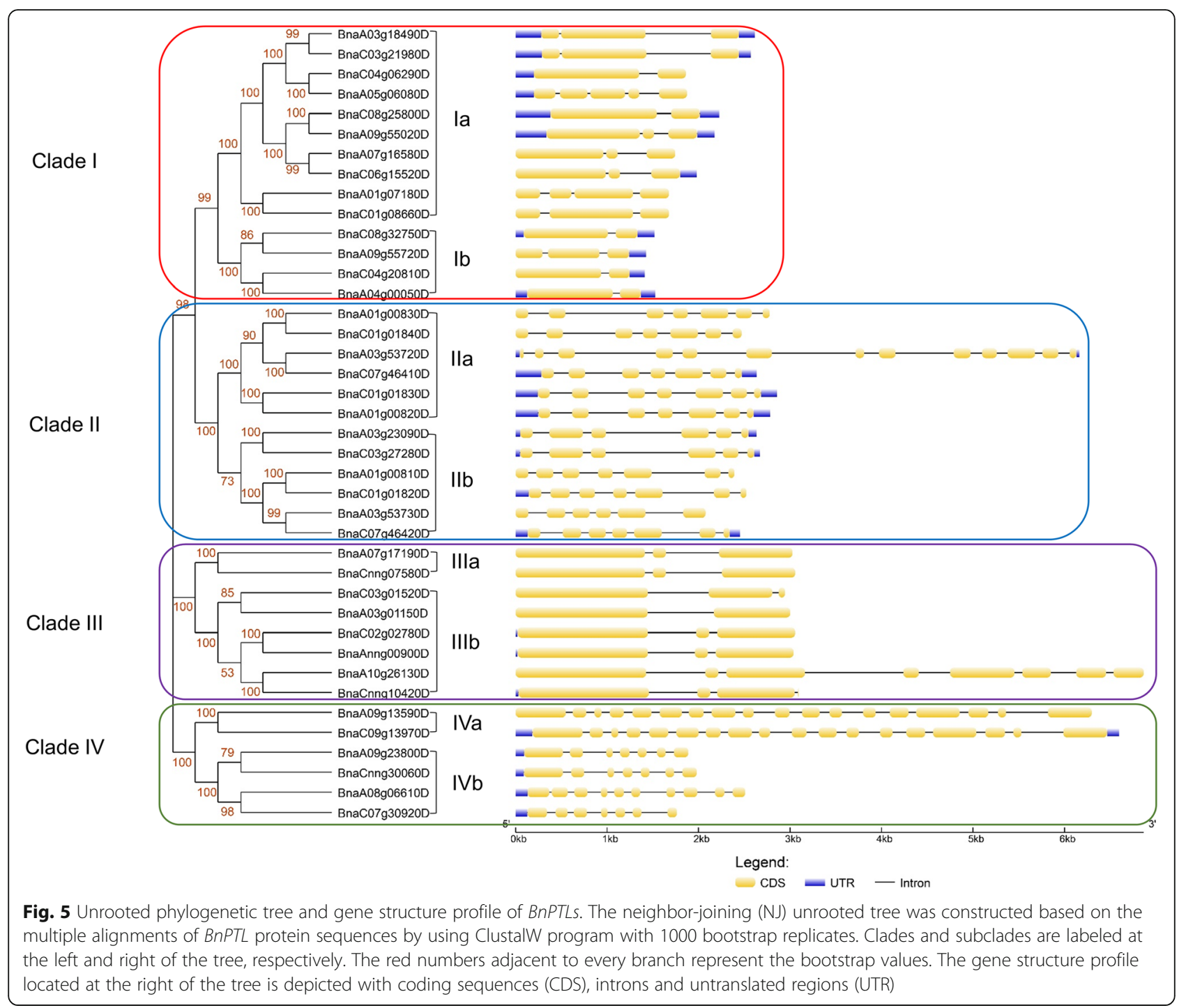

biological significance of the oil content reduction in premature seeds could be the supply of energy and carbon skeleton for the seeds which are going to initiate a next life cycle [12, 40]. Anyway, the decomposition of synthesized lipids during seed maturation eventually led to the reduction of final SOC and downgraded seed quality. Studies showed that Seed Fatty Acid Reducers

Table 2 Catalytic centers of patatin-like lipases in B. napus

\begin{tabular}{|c|c|c|c|}
\hline Clade & Sub-clade & Anion binding box & Esterase box \\
\hline \multirow[t]{2}{*}{ Clade I } & la & LSIDGGGMRG & VAxGSGxGGxxx \\
\hline & $\mathrm{lb}$ & LSIDGGGTIXxx & DIVAGTGIGGXL \\
\hline Clade II & & LSxDGGGxRGXx & DVxxGTSTGGLxx \\
\hline Clade III & & LLLSGGASLGAGAFH & RIIAGSSVGSXX \\
\hline \multirow[t]{2}{*}{ Clade IV } & Iva & ILTMDGGGMKGLA & LICGTSTGGMLA \\
\hline & $\mathrm{IVb}$ & GFSFSAAGLLFPY & DTTPLAGASAGAxVCA \\
\hline
\end{tabular}

Note: The underlined letters indicate regions of the highest homology
(SFARS), namely SFAR1 to SFAR5 belonging to the GDSL family were involved in the decomposition of lipids in developing seeds of Arabidopsis thaliana, and thus reduced SOC and changed fatty acid composition [41]. More recently, BnSFAR1 and BnSFAR4 were successfully silenced by random chemical inducement (Targeting Induced Local Lesions IN Genomes, TILLING), and BnSFAR4 and BnSFAR5 were edited by CRISPRCas9 mediated technology. Mutant lines with up to $12.1 \%$ higher SOC by TILLING and $14.5 \%$ higher SOC by CRISPR-Cas9 were created [15]. In this study, we performed GWAS in a genetic population to associated significant SNPs with acting genes underlying a mapping interval which contained 34 genes. We selected the $P T L$ (BnaC07g30920D) as a candidate gene, which might involve in degrading lipids. We excluded the other 33 genes either from their annotations (Table S2) or by calculating the SNP effects as did with the BnPTL. We 


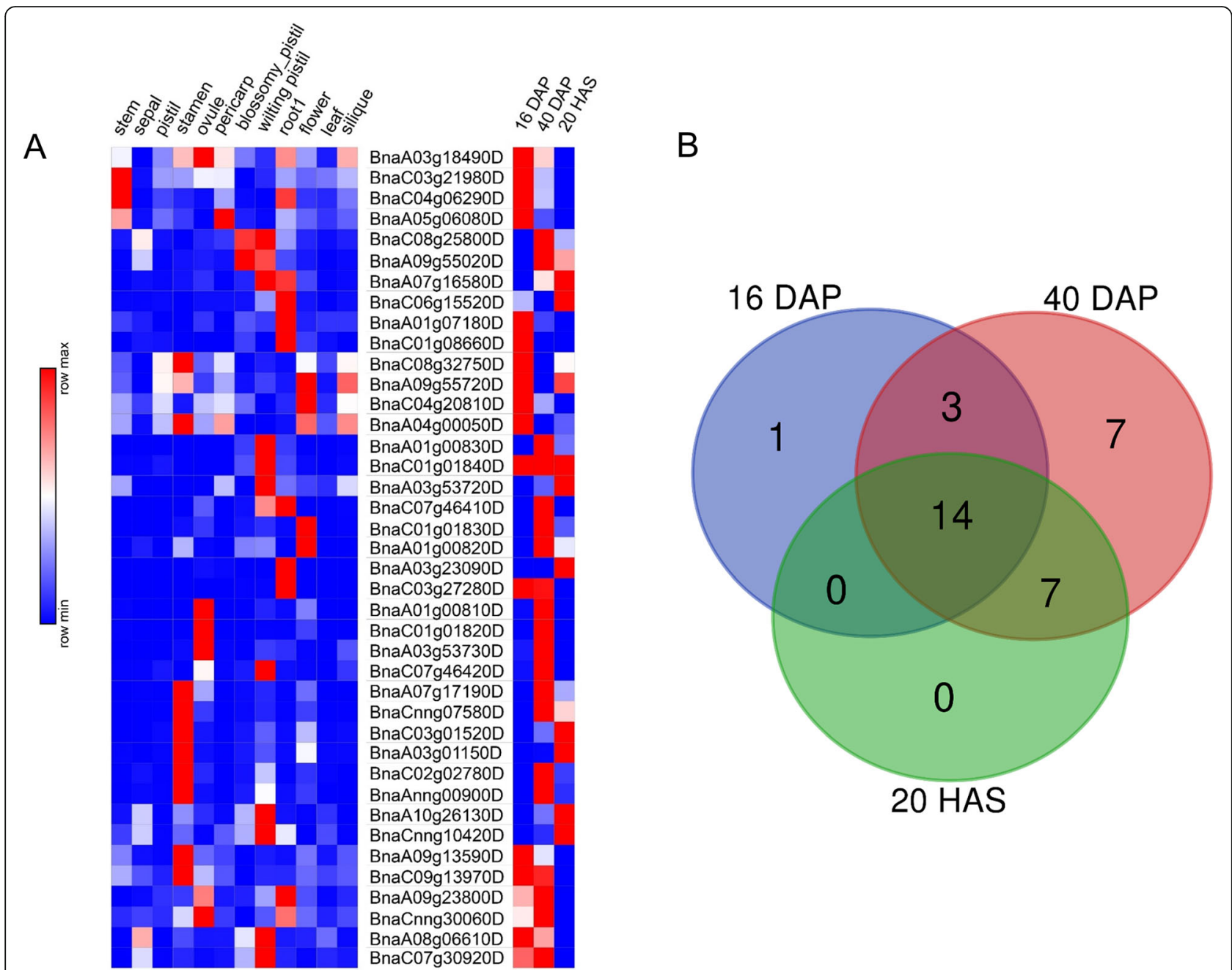

Fig. 6 Expression patterns of BnPTLs based on 2 transcriptome data sets. a The expression heatmap of BnPTLs. The expression heatmap of BnPTLs in 12 different tissues is at the left of the gene IDs and the heatmap at different seed developmental stages is at the right of the gene IDs. The expression levels are shown by the color. b Venn diagram of 40 BnPTLs expressed at different seed developmental stages

show that single nucleotide mutations at BnaC07g30920D, a patatin-like lipase gene, resulted in significant SOC reduction in rapeseed (Fig. 2). Although this PTL and SFARs might have similar biological function, they were likely to be regulated by different upstream regulators. SFARs were regulated by gibberellin signaling [41], whereas PTL remained little understood.

For better exploring potential SOC reducers of the PTL family, in silico analysis was performed to unveil the overall distribution of the genes across the polyploid rapeseed genome. Among 40 BnPTLs, more than 75\% were expressed at 40 DAP (Fig. 6, Table S6), indicating that BnPTLs preferentially function at the late stages of seed development. The time-biased expression pattern of BnPTLs was in line with the period of SOC decrease. There were seven BnPTLs that were exclusively expressed in 40-DAP seeds and 20-HAS germinating seeds (Fig. 6b, Table S6), implying their potential role in oil degradation at these two stages.

As a member of PTL family, SUGAR-DEPENDENT1 (SDP1) is a TAG lipase which is present in many plants such as Arabidopsis thaliana, Brassica napus, Jatropha curcas and Glycine max. Many reports have shown that SDP1 was involved in TAG degradation and the silence of SDP1 could enhance oil content in seeds and other tissues [22, 23, 42-44]. However, the association between the allelic variation of BnSDP1 and SOC was not identified in our GWAS. SOC is a typical quantitative trait that is controlled by the interaction between numerous genes and environmental factors. Our field experiments were carried through in two locations with $400 \mathrm{KM}$ in distance. It could not be excluded that the effect of BnSDP1 could be detected given an environment that could be more different. 
In general, lipases are acyl-hydrolases hydrolyzing the ester bond of mono-, di- and triglycerides (TAGs) into fatty acids and glycerol [45]. Lipases can be divided into three subfamilies according to the structural features of their conserved domains. The largest lipase subfamily contains a conserved signature motif (GXSXG) and their catalytic triad consists of the catalytic serine (S) in this conserved motif, a histidine $(\mathrm{H})$ and either an aspartate (D) or a glutamate (E) located downstream in the sequence $[45,46]$. GDSL-type lipases, which belong to the SGNH hydrolase superfamily, harbor a GDSL sequence motif in sequence block 1 adjacent to the N-terminus instead of the GXSXG motif. The catalytic triad comprises the serine (S) in GDSL motif, the histidine $(\mathrm{H})$ within sequence block 5 and the aspartate (D) 3 amino acids ahead of the $\mathrm{H}(\mathrm{DxxH})[45,47,48]$. Patatin is a lipolytic acyl-hydrolase that is abundant in potato tubers [16]. The biggest structural difference between patatin and other lipases is that its catalytic domain consists of a Ser-Asp dyad. There are 222 GDLS family genes but only 40 PTL family genes in the rapeseed genome (Fig. 4). Enzymes with the patatin catalytic domain, namely PTLs, are widely present in yeast, bacterial, animal and plant. Most of TAG lipases belong to the PTL family, such as TGL3 (yeast), ATGL (mammal) and SDP1 (plant) $[18,45,49]$. The broad substrate specificity of PTLs suggests their multiple biological functions. For example, DENSE AND ERECT PANICLE3 (DEP3), a $P T L$ in rice, was found to play a significant role in the formation of vascular bundles in rice [50]; pPLAIIIS had a marked impact on auxin-responsive cell morphology and organ size in Arabidopsis and B. napus [51]; VdPLP, a PTL in Verticillium dahliae, was involved in cell wall integrity and required for pathogenicity [52]. We classified the 40 BnPTLs in rapeseed into four clades in light of their gene structure and nucleotide sequence similarity. However, the detailed functions of most members of the BnPTL family remain to be explored.

In a recent study, we reported the resequencing of 991 accessions of a world-wide rapeseed (Brassica napus) germplasm collection. A total of 5.56 million SNPs and 1.86 million indels were identified by mapping the reads to a reference genome ('Darmor-bzh') [29]. In this study, we established a gene bank including only 290 accessions based on genetic diversity analysis of the 991 accessions. These 290 accessions stand for more than $97 \%$ genetic polymorphism of the 991 -accessions population in terms of SNPs and InDels, the population was small though. Relative to previous GWASs in rapeseed [29, 30, 53-55], the present study applied a much smaller number of genetic accessions which was easily treated for field replicates. However, the 2,705,480 SNPs, which were very high in number, allow a very powerful identification of tightly associated genes. The results enrich our knowledge about the SOC formation in rapeseed and facilitate the future study in functional characterization of $B n P T L$ genes.

\section{Conclusions}

Patatin-like lipases (PTLs) are widely present in plants and the functions of most PTLs are little understood. In the present study, a BnPTL gene was identified to be associated with SOC using a GWAS on SOC in a collection of 290 core germplasm accessions. A total of 40 $B n P T L$ genes were characterized and divided into four clades, evenly distributed on the A and $\mathrm{C}$ subgenomes of $B$. napus. Moreover, RNA-seq analysis unveiled that BnPTLs were preferentially expressed in reproductive tissues especially maturing seeds. These results enrich our knowledge about the SOC formation in rapeseed and provide more information for future study in functional characterization of BnPTL genes in B. napus.

\section{Methods}

\section{Plant materials and growth conditions}

The 290 core accessions of rapeseed germplasm for GWAS were selected from a worldwide germplasm collection of 991 accessions [29], which were also used to unveil new genes involved in leaf trichome formation in rapeseed in our previous studies [28]. A part of the accessions were acquired from the Leibniz Institute of Plant Genetics and Crop Plant Research (https://gbis. ipk-gatersleben.de/gbis2i/faces/index.jsf) in Gatersleben, Germany, and the rest were from the Provincial Key Laboratory of Crop Gene Resources of Zhejiang University in China. The information about their ID, country origin and SOC of each accession was listed in Table S1. They were grown in the experimental field of Changxing Agricultural Experiment Station of Zhejiang University $\left(30^{\circ} 02^{\prime} \mathrm{N}\right.$ and $\left.119^{\circ} 93^{\prime} \mathrm{E}\right)$ in 2017 and the Experimental Farm of Jinhua Academy of Agricultural Sciences $\left(29^{\circ} 05^{\prime} \mathrm{N}\right.$ and $\left.119^{\circ} 38^{\prime} \mathrm{E}\right)$ in 2018 , respectively.

\section{Seed oil content measurement and phenotypic analysis}

Mature seeds of the 290 core accessions were harvested for SOC measurement. SOCs were determined using near-infrared spectroscopy (ANTARIS II, Thermo Scientific $^{\text {tm }}$, America). Three biological replicates of each accession were measured. Phenotypic analysis including the mean, standard deviation, correlation coefficient, and minimum and maximum values of SOC from 290 accessions were calculated and analyzed using SPSS (http:// www.ibm.com/cn-zh/analytics/spss-statistics-software; V21.0.0.0). Variations in SOC were analyzed by analysis of variance (ANOVA). 


\section{Genome-wide association study}

A total of 2,705,480 high-quality SNPs (MAF $>0.05$, missing rates $<0.5$ ) among the 290 core accessions were extracted and used for GWAS. The K-value, which represents the genetic relations between samples, was calculated by Plink software (http://www.cog-genomics.org/ plink2; V1.9) [56]. TASSEL software (http://www.maize genetics.net/tassel) [57] with an Efficient Mixed-Model Association eXpedited (EMMAX) was used to detect the associations. The $p$-value of each SNP was calculated with $-\log _{10} p>5$ as the suggestive threshold. Seventy-five kilobase sequence regions adjacent to significantly associated SNPs were searched for associated genes. The $\mathrm{R}$ package "LDheatmap" (http://cran.r-project.org/web/ packages/LDheatmap/index.html; V0.99) was used to describe the degree of correlation between significantly associated SNP and candidate genes.

\section{Identification of PTLs in B. napus}

The full genome sequence, protein sequences and their locations of $B$. napus ('Darmor-bzh', V4.1) were retrieved from Brassica napus Genome Browser in GENOSCOPE (http://www.genoscope.cns.fr/brassicanapus/) [58]. Protein sequences were used as queries to search against InterPro database (http://www.ebi.ac.uk/interpro/search/ sequence/; V79.0) [59] with the default parameters. In total, 40 protein sequences with IPR002641 (Patatin-like phospholipase domain) were identified. The distribution of BnPTLs across the rapeseed genome was visualized using MapGene2Chromosome V2.0 (http://mg2c.iask.in/ mg2c_v2.0/).

\section{Search for cis-elements}

We BLAST the nucleotide sequence $5 \mathrm{~Kb}$ upstream to the start codon in the databases PlantProm (http://linux1.soft berry.com/berry.phtml?topic=plantprom \&group $=$ data\& subgroup=plantprom) [60] and AGRIS (https://agrisknowledgebase.org/) [61].

\section{Phylogenetic analysis of BnPTLs and conserved domain search}

Multiple alignments were performed for $B n P T L$ protein sequences using ClustalW of the Molecular Evolutionary Genetics Analysis (MEGAX) program [62]. The unrooted phylogenetic tree was constructed using the neighbor-joining (NJ) method (the Jones-Taylor-Thornton model) with 1000 bootstrap replicates based on the alignment of BnPTL protein sequences in MEGAX. The gene structure and phylogenetic tree were combined using Gene Structure Display Server V2.0 (GSDS 2.0; http://gsds.cbi.pku.edu.cn/) [63]. The conserved domains of BnPTLs were searched based on the previous study [18].

\section{RNA-seq analysis}

Transcriptome data from 12 tissues of the B. napus cultivar 'ZS 11' which was released in the previous study [36] were retrieved from National Center for Biotechnology Information (NCBI; http://www.ncbi.nlm.nih.gov/) (ID: PRJNA394926). DESeq2 R package (http://bioconductor. org/; V3.11) [64] was applied for expression analysis. Transcriptome data of seeds at 16 days after pollination (DAP), 40 DAP and germinating seeds $20 \mathrm{~h}$ after sowing (HAS) were obtained from the previous study [1]. Expression values of these two transcriptome datasets were represented by the number of read counts after calibration. In order to better compare the expression levels in different periods, we also calculated the reads per kilobase per million mapped reads (RPKM) of the latter dataset. The heatmap of BnPTLs was constructed with Morpheus (http://software.broadinstitute.org/morpheus).

\section{Supplementary Information}

The online version contains supplementary material available at https://doi. org/10.1186/s12870-020-02774-w.

Additional file 1: Figure S1. The frequency distribution for SOC of 290 rapeseed accessions in $\mathrm{CX}(\mathrm{A})$ and $\mathrm{JH}(\mathrm{B})$.

Additional file 2: Figure S2. Manhattan plots of GWAS for CX (A) and Jinhua (B). The blue line represents a significant threshold $\left(-\log _{10} p=5\right)$. The black triangle indicates the SNP, ChrC07_35249208, associated with BnaC07g30920D.

Additional file 3: Figure S3. Quantile-quantile (Q-Q) plots of GWAS for $\mathrm{CX}(\mathrm{A})$ and $\mathrm{JH}(\mathrm{B})$. The $\mathrm{Y}$-axis is the observed negative base 10 logarithms of the $P$-values and the $X$-axis is the expected observed negative base 10 logarithms of the $P$-values under the assumption that the $P$-values follow a uniform $(0,1)$ distribution.

Additional file 4: Figure S4. The association of allelic changes in the CDS region and intron of BnaC07g30920D with SOC. ChrC07_35175173 (A) and ChrC07_35175214 (B) were located in introns, while

ChrC07_35175691 (C) and ChrC07_35176144 (D) were located in the CDS region.

Additional file 5: Table S1. The accession codes, names, origins of the 290 genetic materials and their SOC at two experimental sites.

Additional file 6: Table S2. The SNPS associated with SOC on Chr.CO7 and associated gene IDs on Chr.C07.

Additional file 7: Table S3. The SNPs across BnaC07g30920D.

Additional file 8: Table S4. The genome-wide analysis of 40 BnPTLs in rapeseed.

Additional file 9: Table S5. The expression values (indicated by the number of read counts after calibration) of BnPTLs in 12 different tissues of rapeseed.

Additional file 10: Table S6. The expression values (indicated by RPKM and the number of read counts after calibration) of BnPTLs at different seed developmental stages of rapeseed.

\section{Abbreviations}

ANOVA: Analysis of variance; ATGL: Adiponutrin; BnPTL: Brassica napus patatin-like lipase; CDS: Coding sequence; CV: Coefficient of variation; CX: Changxing; DAP: Days after pollination; DEP3: DENSE AND ERECT PANI CLE3; EMMAX: Efficient Mixed-Model Association eXpedited; FA: Fatty acid; GWAS: Genome-wide association study; HAS: Hours after sowing; JH: Jinhua; LD: Linkage disequilibrium; LEC1: LEAFY COTYLEDON1; MEGAX: Molecular Evolutionary Genetics Analysis; NJ: Neighbor-joining; PTL: Patatin-like lipase; Q-Q: Quantile-quantile; QTL: Quantitative trait locus; RPKM: Reads per 
kilobase per million mapped reads; SDP1: SUGAR-DEPENDENT1; SFAR: Seed Fatty Acid Reducer; SNP: Single nucleotide polymorphism; SOC: Seed oil content; TAG: Triacylglycerol; TF: Transcription factor; TILLING: Targeting Induced Local Lesions IN Genomes

\section{Acknowledgements}

We acknowledge Le Zhu, Ying Xu, Mengxin Tu, Xinze Zhao for their helps in the field experiments and the measurement of SOC.

\section{Authors' contributions}

$\sqcup$ and HW conceived the experiments. HW carried through the field experiments and data analyses. QW, HP, TY, MC, XC, DW participated the experiments and data analyses. HW and $\sqcup$ wrote the manuscript. All authors have read and approved the manuscript, and ensure that this is the case.

\section{Funding}

The work was sponsored by Natural Science Foundation of China (Code No. 31961143008 and 31971817) and Jiangsu Collaborative Innovation Centre for Modern Crop Production. These funding bodies financially supported our field experiments, data analyses and personnel allowance.

\section{Availability of data and materials}

The raw reads of the rapeseed accessions used in this study have been deposited in the public database of National Center of Biotechnology Information under SRP155312 (https://www.ncbi.nlm.nih.gov/sra/SRP155312). SNPs among the genetic accessions can be conveniently retrieved at BnaSNPDB (https://bnapus-zju.com/bnasnpdb/). The supporting transcriptome datasets are available at NCBI Bioproject repository, accession number: PRJNA394926.

\section{Ethics approval and consent to participate}

Not applicable.

\section{Consent for publication}

Not applicable.

\section{Competing interests}

The authors declare that they have no competing interests.

\section{Author details}

${ }^{1}$ Institute of Crop Science, Zhejiang University, Yu-Hang-Tang Road 866, Hangzhou 310058, China. ${ }^{2}$ State Key Laboratory of Crop Stress Biology for Arid Areas and College of Agronomy, Northwest A\&F University, Yangling 712100, Shaanxi, China. ${ }^{3}$ Institute of Crop Science, Jinhua Academy of Agricultural Sciences, 828 Shuanglong Nan, Jinhua 321017, China.

Received: 21 August 2020 Accepted: 2 December 2020 Published online: 06 January 2021

\section{References}

1. Zhou L, Wang H, Chen X, Li Y, Hussain N, Cui L, et al. Identification of candidate genes involved in fatty acids degradation at the late maturity stage in Brassica napus based on transcriptomic analysis. Plant Growth Regul. 2017;83:385-96.

2. Xiao Z, Zhang C, Tang F, Yang B, Zhang L, Liu J, et al. Identification of candidate genes controlling oil content by combination of genome-wide association and transcriptome analysis in the oilseed crop Brassica napus. Biotechnol Biofuels. 2019;12:216.

3. Li-Beisson Y, Shorrosh B, Beisson F, Andersson MX, Arondel V, Bates PD, et al. Acyl-lipid metabolism. Arabidopsis Book. 2013;11. https://doi.org/10. 1199/tab.0161

4. Andre C, Froehlich JE, Moll MR, Benning C. A Heteromeric Plastidic pyruvate kinase complex involved in seed oil biosynthesis in Arabidopsis. Plant Cell. 2007;19:2006-22.

5. Andriotis VME, Pike MJ, Schwarz SL, Rawsthorne S, Wang TL, Smith AM. Altered starch turnover in the maternal plant has major effects on Arabidopsis fruit growth and seed composition. Plant Physiol. 2012;160: 1175-86.

6. Baud S, Lepiniec L. Physiological and developmental regulation of seed oil production. Prog Lipid Res. 2010:49:235-49.

7. Ohlrogge J, Browse J. Lipid biosynthesis. Plant Cell. 1995;7:957-70.
8. Roscoe TJ, Lessire R, Puyaubert J, Renard M, Delseny M. Mutations in the fatty acid elongation 1 gene are associated with a loss of $\beta$-ketoacyl-CoA synthase activity in low erucic acid rapeseed. FEBS Lett. 2001;492:107-11.

9. To A, Valon C, Savino G, Guilleminot J, Devic M, Giraudat J, et al. A network of local and redundant gene regulation governs Arabidopsis seed maturation. Plant Cell. 2006;18:1642-51.

10. Chia TYP, Pike MJ, Rawsthorne S. Storage oil breakdown during embryo development of Brassica napus (L.). J Exp Bot. 2005;56:1285-96.

11. Ahc H. Oleosins and oil bodies in seeds and other organs. Plant Physiol. 1996;110:1055-61.

12. Graham IA. Seed storage oil mobilization. Annu Rev Plant Biol. 2008;59:11542.

13. Baker A, Graham IA, Holdsworth M, Smith SM, Theodoulou FL. Chewing the fat: beta-oxidation in signalling and development. Trends Plant Sci. 2006;11: 124-32.

14. Eastmond PJ, Graham IA. Re-examining the role of the glyoxylate cycle in oilseeds. Trends Plant Sci. 2001;6:72-8

15. Karunarathna NL, Wang H, Harloff H-J, Jiang L, Jung C. Elevating seed oil content in a polyploid crop by induced mutations in SEED FATTY ACID REDUCER genes. Plant Biotechnol J. 2020; https://doi.org/10.1111/pbi.13381.

16. Galliard T. The enzymic breakdown of lipids in potato tuber by phospholipid- anld galactolipid-acm, hydrolase activities and by lipoxygenase. Phytochemistry. 1970;9:1725-34.

17. Rydel TJ, Williams JM, Krieger E, Moshiri F, Stallings WC, Brown SM, et al. The crystal structure, mutagenesis, and activity studies reveal that Patatin is a lipid acyl hydrolase with a Ser-asp catalytic dyad. Biochemistry. 2003;42: 6696-708.

18. Scherer GFE, Ryu SB, Wang X, Matos AR, Heitz T. Patatin-related phospholipase a: nomenclature, subfamilies and functions in plants. Trends Plant Sci. 2010;15:693-700.

19. Wilson SK, Knoll L. Patatin-like phospholipases in microbial infections with emerging roles in fatty acid metabolism and immune regulation by Apicomplexa. Mol Microbiol. 2018;107:34-46.

20. Yang W, Devaiah SP, Pan X, Isaac G, Welti R, Wang X. AtPLAl is an acyl hydrolase involved in basal Jasmonic acid production and Arabidopsis resistance to Botrytis cinerea. J Biol Chem. 2007;282:18116-28.

21. Dhondt S, Gouzerh G, Müller A, Legrand M, Heitz T. Spatio-temporal expression of patatin-like lipid acyl hydrolases and accumulation of jasmonates in elicitor-treated tobacco leaves are not affected by endogenous levels of salicylic acid. Plant J. 2002;32:749-62.

22. Eastmond PJ. SUGAR-DEPENDENT1 encodes a Patatin domain triacylglycerol lipase that initiates storage oil breakdown in germinating Arabidopsis seeds. Plant Cell. 2006;18:665-75.

23. Kelly AA, Shaw E, Powers SJ, Kurup S, Eastmond PJ. Suppression of the SUGAR-DEPENDENT1 triacylglycerol lipase family during seed development enhances oil yield in oilseed rape (Brassica napus L.). Plant Biotechnol J. 2013;11:355-61.

24. Jiang C, Shi J, Li R, Long Y, Wang H, Li D, et al. Quantitative trait loci that control the oil content variation of rapeseed (Brassica napus L.). Theor Appl Genet. 2014;127:957-68.

25. Chen G, Geng J, Rahman M, Liu X, Tu J, Fu T, et al. Identification of QTL for oil content, seed yield, and flowering time in oilseed rape (Brassica napus). Euphytica. 2010;175:161-74.

26. Biljana B, Vesna K, Biljana G. Fatty acid composition of seed oil obtained from different canola varieties. Farm Glas. 2015:71:1-7.

27. Zhao J, Dimov Z, Becker HC, Ecke W, Möllers C. Mapping QTL controlling fatty acid composition in a doubled haploid rapeseed population segregating for oil content. Mol Breed. 2008;21:115-25.

28. Xuan L, Yan T, Lu L, Zhao X, Wu D, Hua S, et al. Genome-wide association study reveals new genes involved in leaf trichome formation in polyploid oilseed rape (Brassica napus L.). Plant Cell Environ. 2020;43:675-91.

29. Wu D, Liang Z, Yan T, Xu Y, Xuan L, Tang J, et al. Whole-genome Resequencing of a worldwide collection of rapeseed accessions reveals the genetic basis of ecotype divergence. Mol Plant. 2019;12:30-43.

30. Wang J, Xian X, Xu X, Qu C, Lu K, Li J, et al. Genome-wide association mapping of seed coat color in Brassica napus. J Agric Food Chem. 2017;65: 5229-37.

31. Du X, Huang G, He S, Yang Z, Sun G, Ma X, et al. Resequencing of 243 diploid cotton accessions based on an updated a genome identifies the genetic basis of key agronomic traits. Nat Genet. 2018;50:796-802. 
32. Zhou Z, Jiang Y, Wang Z, Gou Z, Lyu J, Li W, et al. Resequencing 302 wild and cultivated accessions identifies genes related to domestication and improvement in soybean. Nat Biotechnol. 2015;33:408-14.

33. Meuwissen THE, Goddard ME. Fine mapping of quantitative trait loci using linkage disequilibria with closely linked marker loci. Genetics. 2000;155:421-30.

34. Qu C, Jia L, Fu F, Zhao H, Lu K, Wei L, et al. Genome-wide association mapping and identification of candidate genes for fatty acid composition in Brassica napus L. using SNP markers. BMC Genomics. 2017;18:232.

35. Slatkin M. Linkage disequilibrium - understanding the evolutionary past and mapping the medical future. Nat Rev Genet. 2008;9:477-85.

36. Sun F, Fan G, Hu Q, Zhou Y, Guan M, Tong C, et al. The high-quality genome of Brassica napus cultivar 'ZS11' reveals the introgression history in semi-winter morphotype. Plant J. 2017;92:452-68.

37. Liu F, Xia Y, Wu L, Fu D, Hayward A, Luo J, et al. Enhanced seed oil content by overexpressing genes related to triacylglyceride synthesis. Gene. 2015; 557:163-71.

38. Wei W, Li G, Jiang X, Wang Y, Ma Z, Niu Z, et al. Small RNA and degradome profiling involved in seed development and oil synthesis of Brassica napus. PLoS One. 2018;13:e0204998.

39. Murphy DJ, Cummins I, Kang AS. Synthesis of the major oil-body membrane protein in developing rapeseed (Brassica napus) embryos. Integration with storage-lipid and storage-protein synthesis and implications for the mechanism of oil-body formation. Biochem J. 1989:258:285-93.

40. Theodoulou FL, Eastmond PJ. Seed storage oil catabolism: a story of give and take. Curr Opin Plant Biol. 2012;15:322-8.

41. Chen M, Du X, Zhu Y, Wang Z, Hua S, Li Z, et al. Seed Fatty Acid Reducer acts downstream of gibberellin signalling pathway to lower seed fatty acid storage in Arabidopsis. Plant Cell Environ. 2012;35:2155-69.

42. Kanai M, Yamada T, Hayashi M, Mano S, Nishimura M. Soybean (Glycine max L.) triacylglycerol lipase GmSDP1 regulates the quality and quantity of seed oil. Sci Rep. 2019;9:8924.

43. Kim MJ, Yang SW, Mao H-Z, Veena SP, Yin J-L, Chua N-H. Gene silencing of Sugar-dependent 1 (JCSDP1), encoding a patatin-domain triacylglycerol lipase, enhances seed oil accumulation in Jatropha curcas. Biotechnol Biofuels. 2014;7:36

44. Nobusawa T, Yamakawa-Ayukawa K, Saito F, Nomura S, Takami A, Ohta H. A homolog of Arabidopsis SDP1 lipase in Nannochloropsis is involved in degradation of de novo-synthesized triacylglycerols in the endoplasmic reticulum. Biochim Biophys Acta Mol Cell Biol Lipids. 2019;1864:1185-93.

45. Kelly AA, Feussner I. Oil is on the agenda: lipid turnover in higher plants. Biochim Biophys Acta. 1861;2016:1253-68.

46. Casas-Godoy L, Duquesne S, Bordes F, Sandoval G, Marty A. Lipases: an overview. Methods Mol Biol. 2012;861:3-30.

47. Upton C, Buckley JT. A new family of lipolytic enzymes? Trends Biochem Sci. 1995;20:178-9

48. Akoh CC, Lee G-C, Liaw Y-C, Huang T-H, Shaw J-F. GDSL family of serine esterases/lipases. Prog Lipid Res. 2004;43:534-52.

49. Quettier A-L, Eastmond PJ. Storage oil hydrolysis during early seedling growth. Plant Physiol Biochem. 2009;47:485-90.

50. Qiao Y, Piao R, Shi J, Lee S-I, Jiang W, Kim B-K, et al. Fine mapping and candidate gene analysis of dense and erect panicle 3, DEP3, which confers high grain yield in rice (Oryza sativa L.). Theor Appl Genet. 2011;122:143949.

51. Dong Y, Li M, Zhang P, Wang X, Fan C, Zhou Y. Patatin-related phospholipase pPLAIll $\delta$ influences auxin-responsive cell morphology and organ size in Arabidopsis and Brassica napus. BMC Plant Biol. 2014;14:332.

52. Qi X, Li X, Guo H, Guo N, Cheng H. VdPLP, a Patatin-like phospholipase in Verticillium dahliae, is involved in cell wall integrity and required for pathogenicity. Genes. 2018;9:162.

53. Arifuzzaman M, Oladzadabbasabadi A, McClean P, Rahman M. Shovelomics for phenotyping root architectural traits of rapeseed/canola (Brassica napus L.) and genome-wide association mapping. Mol Gen Genomics. 2019;294: 985-1000.

54. He Y, Wu D, Wei D, Fu Y, Cui Y, Dong H, et al. GWAS, QTL mapping and gene expression analyses in Brassica napus reveal genetic control of branching morphogenesis. Sci Rep. 2017;7:15971.

55. Lu K, Wei L, Li X, Wang Y, Wu J, Liu M, et al. Whole-genome resequencing reveals Brassica napus origin and genetic loci involved in its improvement Nat Commun. 2019;10:1154.
56. Purcell S, Neale B, Todd-Brown K, Thomas L, Ferreira MAR, Bender D, et al. PLINK: a tool set for whole-genome association and population-based linkage analyses. Am J Hum Genet. 2007;81:559-75.

57. Bradbury PJ, Zhang Z, Kroon DE, Casstevens TM, Ramdoss Y, Buckler ES TASSEL: software for association mapping of complex traits in diverse samples. Bioinformatics. 2007;23:2633-5.

58. Chalhoub B, Denoeud F, Liu S, Parkin IAP, Tang H, Wang X, et al. Early allopolyploid evolution in the post-Neolithic Brassica napus oilseed genome. Science. 2014;345:950-3.

59. Apweiler R, Attwood TK, Bairoch A, Bateman A, Birney E, Biswas M, et al. The InterPro database, an integrated documentation resource for protein families, domains and functional sites. Nucleic Acids Res. 2001;29:37-40.

60. Shahmuradov I, Gammerman A, Hancock J, Bramley P, Solovyev V. PlantProm: a database of plant promoter sequences. Nucleic Acids Res. 2003;31:114-7.

61. Davuluri RV, Sun H, Palaniswamy SK, Matthews N, Molina C, Kurtz M, et al. AGRIS: Arabidopsis gene regulatory information server, an information resource of Arabidopsis cis-regulatory elements and transcription factors. BMC Bioinformatics. 2003;4:25.

62. Kumar S, Stecher G, Li M, Knyaz C, Tamura K. MEGA X: Molecular evolutionary genetics analysis across computing platforms. Mol Biol Evol. 2018:35:1547-9.

63. Hu B, Jin J, Guo A-Y, Zhang H, Luo J, Gao G. GSDS 2.0: an upgraded gene feature visualization server. Bioinformatics. 2015:31:1296-7.

64. Love Ml, Huber W, Anders S. Moderated estimation of fold change and dispersion for RNA-seq data with DESeq2. Genome Biol. 2014;15:550.

\section{Publisher's Note}

Springer Nature remains neutral with regard to jurisdictional claims in published maps and institutional affiliations.

Ready to submit your research? Choose BMC and benefit from:

- fast, convenient online submission

- thorough peer review by experienced researchers in your field

- rapid publication on acceptance

- support for research data, including large and complex data types

- gold Open Access which fosters wider collaboration and increased citations

- maximum visibility for your research: over $100 \mathrm{M}$ website views per year

At $\mathrm{BMC}$, research is always in progress.

Learn more biomedcentral.com/submissions 